\title{
Japanese Intake of Flavonoids and Isoflavonoids from Foods
}

\author{
Mitsuru Kimira ${ }^{1}$, Yusuke Arai ${ }^{1}$, Kayako Shimoi ${ }^{2}$, and Shaw Watanabe ${ }^{1}$
}

The intake of flavonoids and isoflavonoids was estimated based upon a preliminary database of $\mathbf{4 0}$ food items, covering at least $\mathbf{8 0 \%}$ of total food consumption. Fifty volunteer women in "ICity" recorded the weight of all dietary intake for 3 days in September 1996, and received a health check-up, as well as laboratory examination. The data was analyzed in relation to the various food factors.

Average daily intake per capita of flavonoids was as follows: $4.9 \mathrm{mg}$ kaempferol, $8.3 \mathrm{mg}$ quercetin, $1.5 \mathrm{mg}$ rutin, $0.6 \mathrm{mg}$ myricetin, $0.3 \mathrm{mg}$ luteolin, $0.01 \mathrm{mg}$ myricitrin, $0.4 \mathrm{mg}$ fisetin, and $0.3 \mathrm{mg}$ eriodictyol. Total intake from vegetables and fruits was less than $10 \mathrm{mg} .16 .2 \mathrm{mg}$ (range: $3.18-35.61 \mathrm{mg})$ and $23.27 \mathrm{mg}(4.62-52.12 \mathrm{mg})$ of isoflavones, such as daidzein and genistein, respectively, were taken per day, and total isoflavone intake was $39.46 \mathrm{mg}(7.80-87.73 \mathrm{mg})$.

Chief component analysis on ingested vitamins, flavonoids and isoflavonoids was carried out. Factor 1 was mainly composed of flavonoids and antioxidant vitamins. Factor 1 was positively associated with age and the level of HDL cholesterol and negatively related to the level of triglycerides. Factor 2, which was mainly composed of isoflavonoids, was positively associated with creatinine and uric acid levels. So far, these factors did not show a significant association with bone density and other health indices, such as BMI and blood pressure.

J Epidemiol, $1998 ; 8$ : 168-175.

Non-nutrient food chemicals (factors) carry on various pharmacological activities in the human body. Antioxidant properties of flavonoids, such as quercetin and kaempferol, are considered to attribute to the low risk of coronary heart disease in western countries ${ }^{1,2,3)}$. Japanese consume a lot of soy bean products, containing phytoestrogens, such as daidzein and genistein, which may lead to the low incidence of estrogenrelated cancers ${ }^{4,5}$ ). More than 600 food factors in vegetables are considered to be effective for cancer prevention through various influences on the metabolism inside the body. Accurate estimation of these chemical intake becomes important, but an appropriate database for Japanese foods has not been available. A preliminary database was built in this study to estimate the amount of flavonoids and isoflavonoids ingested by Japanese.

\section{MATERIALS AND METHODS}

Intake of food factors (phytochemicals) was calculated from the field study in "I-City" of Iwate Prefecture. This field study has been continued since 1980 under collaboration with the local government. In early September, 1995, volunteer housewives in fifty families were asked to record the weight of all food intake and cooking methods for 3 consecutive days. Their age distribution was from 32 to 68 years of age. The dietary records were checked through interviews by trained dieticians on the fourth day when the physical examinations were scheduled. Samples of $10 \mathrm{ml}$ of blood were also collected from each volunteer for blood chemistry analysis.

Blood samples were measured for RBC, WBC, $\mathrm{Ht}, \mathrm{Hb}$, GOT, GPT, $\gamma$-GTP, AlP, total protein, albumin, A/G ratio,

Received November 24, 1997 ; accepted March 25, 1998.

'Laboratory of Nutrition and Epidemiology, Department of Applied Bioscience, Tokyo University of Agriculture.

${ }^{2}$ Department of Food Science, Shizuoka Prefectural University.

Address for correspondence : Shaw Watanabe, Tokyo University of Agriculture, 1-1-1, Sakuragaoka, Setagaya, Tokyo 156-0054, Japan. 
creatinine, uric acid, total cholesterol, HDL cholesterol, triglycerides, choline esterase, total bilirubin and blood sugar. All the above blood analysis was performed by the Iwate Health Foundation.

Physical examinations, such as height, body weight, blood pressure, and bone density determined by ultrasound (A-1000, Lunar Co. Ltd., Tokyo), were carried out at the time of the health check-up. The number of steps per day, as an index of moving, were also measured by a step recorder (Yamasa Watch Co. Ltd.,). Physical and blood clinical data were summarized in tablel, 2.

\begin{tabular}{|c|c|c|c|c|}
\hline Variable & Mean & S.D. & Min & Max \\
\hline Age (years) & 53.0 & 9.7 & 28.0 & 71.0 \\
\hline Height (cm) & 153.2 & 4.8 & 140.5 & 164.5 \\
\hline Weight (kg) & 53.3 & 7.8 & 41.0 & 81.0 \\
\hline Body Mass Index & 22.8 & 3.2 & 16.3 & 32.5 \\
\hline Systolic blood pressure (mmHg) & 124 & 17 & 90 & 172 \\
\hline Diastolic blood pressure ( $\mathrm{mmHg}$ ) & 77 & 12 & 50 & 100 \\
\hline Bone density & 73.4 & 12.7 & 54.0 & 106.0 \\
\hline
\end{tabular}

\begin{tabular}{|c|c|c|c|c|}
\hline Variable & Mean & S.D. & Min & Max \\
\hline Red Blood Cell $\left(10^{4} / \mu \mathrm{L}\right)$ & 418.4 & 31.2 & 312.0 & 502.0 \\
\hline Hemoglobin (\%) & 12.6 & 1.1 & 9.1 & 15.1 \\
\hline White Blood Cell $\left(10^{2} / \mu \mathrm{L}\right)$ & 61.9 & 12.8 & 33.0 & 88.0 \\
\hline Hematocrit (g/dL) & 38.9 & 2.9 & 31.7 & 46.1 \\
\hline GOT (U/L) & 21 & 5 & 13 & 33 \\
\hline GPT (U/L) & 18 & 8 & 8 & 53 \\
\hline $\mathrm{ALP}(\mathrm{U} / \mathrm{L})$ & 159 & 37 & 88 & 251 \\
\hline Cholinesterase ( $\triangle \mathrm{pH})$ & 0.90 & 0.19 & 0.51 & 1.70 \\
\hline$\gamma-\mathrm{GTP}(\mathrm{U} / \mathrm{L})$ & 20 & 10 & 8 & 43 \\
\hline Total Bilinubin (mg/dL) & 0.57 & 0.23 & 0.30 & 1.20 \\
\hline Total Protein (g/dL) & 7.4 & 0.4 & 6.6 & 8.3 \\
\hline Albumin $(\mathrm{g} / \mathrm{dL})$ & 4.5 & 0.2 & 3.4 & 4.9 \\
\hline Albumin Globulin Ratio & 1.54 & 0.18 & 1.05 & 1.95 \\
\hline Serum total cholesterol (mg/dL) & 208.5 & 38.7 & 121.0 & 295.0 \\
\hline HDL cholesterol (mg/dL) & 57.7 & 14.0 & 30.0 & 90.0 \\
\hline Triglyceride (mg/dL) & 117.2 & 56.9 & 34.0 & 264.0 \\
\hline Glucose (mg/dL) & 104.0 & 21.8 & 57.0 & 154.0 \\
\hline Creatinine (mg/dL) & 0.93 & 0.19 & 0.8 & 2.1 \\
\hline Uric Acid (mg/dl) & 4.2 & 1.0 & 2.8 & 7.4 \\
\hline
\end{tabular}

The intake of flavonoids and isoflavonoids was estimated by calculation from the database. Our own database of flavonoids

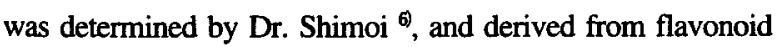
data in foods. Optimization of a quantitative HPLC determination was employed for measuring flavonoids ". Quercetin, kaempferol, myricetin, fisetin, eriodictyol and luteolin were measured by freeze-dried vegetables and fruits after acid hydrolysis of the parent glycosides. In some foods containing rutin and myricitrin, such as bean sprouts, grapefruits, moroheiya, onion, tomato and green tea, methanol extraction yielded more flavonoids; but we employed a value for acid hydrolyzates, because of the more plausible similarities in cooking and absorption into the body. Isoflavonoid value in foods was also measured by the HPLC with a diode array detector and gas chromatography-mass spectrometry after methanol extraction and hydrolysis, according to the modified method of Adlercreut $z^{2}$. A database was made from these values (Table 3).

Each recorded food item was coded, and consumption dose and frequency were summarized. Food nutrients were calculated by using the Standard Tables of Food Composition in Japan (4th revised edition). In addition, values of each consumed food item was multiplied with the flavonoids-isoflavonoids content table (Table 1).

Association with the above described variables was tested in various ways. Principle component analysis was done for antioxidant phytochemicals and vitamins, and association with various health indices was searched. All statistical analysis was performed by the SPSS program package (Ver.7.5.1) after making a database on an IBM computer.

\section{RESULTS}

Food intake of the volunteers met the recommended dietary allowance for Japanese (Fig. 1). Average energy intake was $1886 \mathrm{kcal}$, protein intake $80.2 \mathrm{~g}$, fat intake $56.2 \mathrm{~g}$, and carbohydrate $256.7 \mathrm{~g}$. Vitamin intake exceeded $150 \%$ in most women.

Food composition of the volunteers is shown in Table 4. About one third was vegetables and fruits. Fifty five vegetables (15 species), 6 tuber crops ( 5 species), 5 seeds ( 5 species), and 4 crops were consumed during the 3 days (Table 5). On average, they consumed $365.7 \mathrm{~g}$ of vegetables, $155.5 \mathrm{~g}$ of fruits, $217.6 \mathrm{~g}$ of cereal, and $58.0 \mathrm{~g}$ of potatoes. Per capita consumption of rice, noodles and bread for one day was $203.8 \mathrm{~g}$. Pulse crops (legumes) was $84.5 \mathrm{~g}$, tuber crops was $58.9 \mathrm{~g}$, and nuts and seeds were $2.3 \mathrm{~g}$. Oil consumption was $15.2 \mathrm{~g}$ - most of which was plant oil. The older age group consumed more vegetables than the younger generation. Meat and milk consumption was less in the older age group.

Concerning vitamin intake, antioxidant vitamin $C$ equaled $117 \pm 47 \mathrm{mg} /$ day (mean \pm S.D.), tocopherol equaled $21.15 \pm$ $5.48 \mathrm{mg} /$ day, and carotenoids equaled $3.35 \pm 1.48 \mathrm{mg} /$ day (Table 6). Kaempferol, quercetin, myricetin and rutin were common flavonoids derived from the vegetables. Average intake of these flavonoids was $16.2 \mathrm{mg} /$ day (range 3.7-48.3 $\mathrm{mg}$ ). A breakdown of the flavonoids showed the $4.91 \mathrm{mg}$ kaempferol, $8.28 \mathrm{mg}$ quercetin, $1.50 \mathrm{mg}$ rutin, $0.57 \mathrm{mg}$ myricetin, $0.28 \mathrm{mg}$ luteolin, $0.01 \mathrm{mg}$ myricitrin, $0.39 \mathrm{mg}$ fisetin, and $0.27 \mathrm{mg}$ eriodictyol per day per capita. Quercetin was ingested through the following foods: onions (78.6\%), moroheiya $(14.0 \%)$, green tea $(1.4 \%)$, and oranges $(0.36 \%)$. Foods providing kaempferol were apples $(4.1 \%)$, onions (5.5\%) and carrots $(0.2 \%)$.

Tofu, miso (fermented soy bean) and natto were main foods containing rich isoflavones, and average intake of total agly- 
Table 3. Amount of Flavonoids and Isoflavonoids in Foods.

\begin{tabular}{|c|c|c|c|c|c|c|c|c|c|c|c|}
\hline Food Name & $\mathrm{Da}$ & $\mathrm{Ge}$ & Mytrin & $\mathbf{R u}$ & Mytrin & $\mathrm{Fi}$ & Eri & $\mathrm{Qu}$ & Lulin & $\mathbf{K a}$ & Flav total \\
\hline Kidney Bean & - & - & nd & 4.67 & 11.10 & nd & nd & 12.60 & 10.10 & 18.60 & 57.07 \\
\hline Green Soybean & - & - & nd & nd & nd & nd & nd & 0.30 & nd & 12.30 & 12.60 \\
\hline Soybean seed & 846.20 & 1106.80 & - & - & - & - & - & - & - & - & 1953.00 \\
\hline Soyflour & 654.20 & 1122.60 & - & - & - & - & - & - & - & - & 1777.30 \\
\hline Bean Sprout & - & - & 1.29 & 7.78 & nd & nd & 43.70 & 1.47 & nd & 3.26 & 57.50 \\
\hline Tofu (Momen) & 169.00 & 253.00 & nd & nd & nd & nd & nd & nd & nd & 11.90 & 433.90 \\
\hline Silken tofu (kinu) & 151.00 & 225.00 & - & - & - & - & - & - & - & - & 376.00 \\
\hline Packed Tofu & 220.00 & 294.00 & - & - & - & - & - & - & - & - & 514.00 \\
\hline Baked Tofu & 175.00 & 256.00 & - & - & - & - & - & - & - & - & 431.00 \\
\hline Fried Tofu & 335.00 & 401.00 & - & - & - & - & - & - & - & - & 736.00 \\
\hline Grilled Tofu & 118.00 & 317.00 & - & - & - & - & - & - & - & - & 435.00 \\
\hline $\begin{array}{l}\text { Ganmodoki } \\
\text { (Mix Fried Tofu) }\end{array}$ & 336.00 & 412.00 & - & - & - & - & - & - & - & - & 748.00 \\
\hline Natto & 277.20 & 327.00 & - & - & - & - & - & - & - & - & 604.20 \\
\hline $\begin{array}{l}\text { Soybean paste } \\
\text { (White) }\end{array}$ & 116.00 & 172.00 & - & - & - & - & - & - & - & - & 288.00 \\
\hline $\begin{array}{l}\text { Soybean paste } \\
\text { (Red) }\end{array}$ & 179.00 & 278.00 & - & - & - & - & - & - & - & - & 457.00 \\
\hline Potato & - & - & nd & nd & nd & nd & nd & nd & nd & 23.40 & 23.40 \\
\hline Tomato & - & - & nd & 3.38 & nd & 0.12 & nd & 1.58 & nd & 7.50 & 12.58 \\
\hline Green pepper & - & - & nd & nd & nd & nd & nd & 14.10 & 14.70 & 3.17 & 31.97 \\
\hline Eggplant & - & - & nd & nd & nd & nd & nd & 1.58 & nd & nd & 1.58 \\
\hline Carrot & - & - & nd & 0.02 & nd & nd & nd & nd & nd & 15.30 & 15.32 \\
\hline Parsley & - & - & nd & nd & 216.00 & nd & nd & 7.03 & 3.09 & 45.10 & 271.22 \\
\hline Radish & - & - & nd & 0.55 & nd & nd & nd & nd & nd & 3.36 & 3.91 \\
\hline Cabbage & - & - & nd & nd & nd & nd & nd & nd & nd & 7.20 & 7.20 \\
\hline Broccoli & - & - & nd & 0.56 & 7.05 & nd & nd & 9.77 & nd & 16.10 & 33.48 \\
\hline Moroheiya & - & - & nd & 99.40 & 19.30 & nd & nd & 154.00 & nd & 118.00 & 390.70 \\
\hline Spinach & - & - & nd & 0.24 & 37.50 & nd & nd & nd & 7.13 & nd & 44.87 \\
\hline Lettuce & - & - & 1.23 & 1.24 & nd & nd & nd & 4.78 & 5.23 & nd & 12.48 \\
\hline Onion & - & - & nd & 2.37 & 2.95 & 4.78 & nd & 33.70 & 1.91 & 14.09 & 59.80 \\
\hline Lotus Root & - & - & nd & nd & 5.89 & 5.80 & nd & 4.41 & 3.61 & 7.57 & 27.28 \\
\hline Cucumber & - & - & nd & 0.94 & nd & 0.14 & nd & nd & nd & 7.61 & 8.69 \\
\hline Kiwi fruit & - & - & nd & nd & nd & 2.03 & nd & 2.07 & nd & 30.60 & 34.70 \\
\hline Water Melon & - & - & nd & nd & nd & nd & nd & nd & nd & 18.10 & 18.10 \\
\hline Orange & - & - & nd & nd & 14.40 & nd & nd & 17.50 & 0.96 & 31.50 & 64.36 \\
\hline Peach & - & - & nd & 0.54 & nd & 0.58 & nd & 1.08 & nd & 6.54 & 8.74 \\
\hline Apple & - & - & nd & nd & nd & 26.90 & nd & 5.27 & nd & 26.70 & 58.87 \\
\hline Persimmon & - & - & nd & nd & 10.60 & 10.50 & nd & nd & 1.43 & nd & 22.53 \\
\hline Grape & - & - & nd & nd & nd & 3.93 & nd & nd & nd & 16.80 & 20.73 \\
\hline Strawberry & - & - & nd & 27.10 & nd & 160.00 & nd & 6.91 & nd & 19.40 & 213.41 \\
\hline Green tea (Infusion) & - & - & nd & 3.12 & nd & nd & nd & 1.10 & nd & 0.60 & 4.82 \\
\hline
\end{tabular}

Da: Daidzein Ge: Genistein Mytrin : Myricitrin Ru : rutin Mytin : Myricetin

Fi: Ficetin Eri : Eriodictyol Qu:Quercetin Lu:Luteorin Ka: Kaempferol

nd : not detected 
cones of genistein and daidzein was 39.46 (range 7.80-87.73 $\mathrm{mg} /$ day). Attributable rates of genistein were tofu (49.6\%), soybean paste $(20.9 \%)$, natto $(14.7 \%)$ and cooked soybean $(14.8 \%)$. Foods containing daidzein were similar. Intake of flavonoids, isoflavonoids, vitamins and carotenoids are summarized in Table 6.

Intake of flavonoids showed significantly positive correlation with vitamin $C$ intake, and carotene intake (Fig. 2ab). Intake of isoflavonoids was associated with tocopherol intake (Fig. 2c).

Principle component analysis of these factors yielded several groups (Table 7). Factor 1 was mainly composed of retinol, caroteinoid, vitamin $\mathrm{C}$, rutin, myricetin and kaempferol, suggesting a rich vegetable intake; factor 2 was composed of daidzein, genistein, myricitrin, eriodictyol, and tocopherol, suggesting a rich pulse intake; factor 3 showed positive direction for myricitrin and eriodictyol and negatively for daidzein and genistein. Association between these factors and food items is shown in Table 8. Factor 1 was significantly associated with vegetables, fish and potatoes; factor 2 with legumes and fat and oil; factor 3 showed positive correlation with noodles and milk, and negatively with legumes. Association between the above factors and physical and laboratory data of

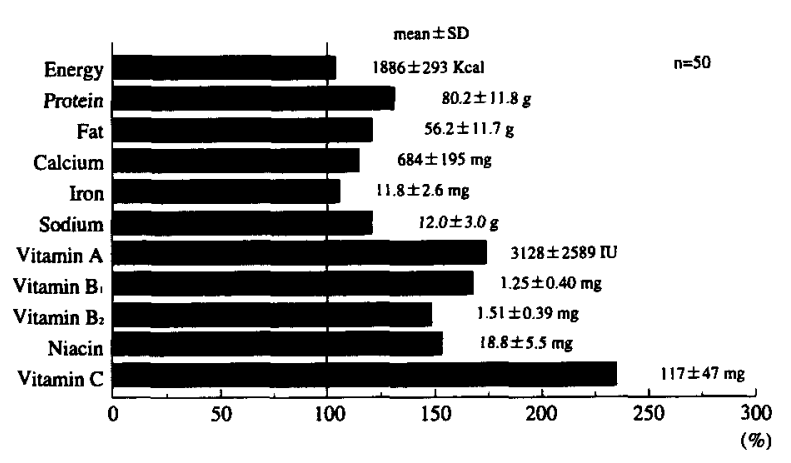

Figure 1. Rate of Food intake and Required dietary allowance

\begin{tabular}{lrrrr}
\multicolumn{5}{c}{ Table 4. Intake of Foods in 13 Food Categories. } \\
& & & $\mathrm{N}=50$ & g/day capita \\
\hline \multicolumn{1}{c}{ Variable } & Mean & S.D. & \multicolumn{1}{c}{ Min } & Max \\
\hline Cereals & 217.6 & 52.1 & 109.3 & 337.7 \\
Nuts and Seeds & 2.3 & 4.0 & 0.0 & 20.0 \\
Potatoes & 58.0 & 45.4 & 0.0 & 256.7 \\
Fats and Oils & 15.3 & 7.2 & 1.3 & 33.2 \\
Legumes & 84.5 & 41.2 & 20.7 & 198.3 \\
Fruits & 155.5 & 121.1 & 0.0 & 598.3 \\
Vegetables & 365.7 & 141.6 & 117.3 & 764.0 \\
Fungi & 12.2 & 14.8 & 0.0 & 83.3 \\
Algae & 5.9 & 7.9 & 0.1 & 37.4 \\
Fishes & 96.5 & 35.6 & 38.7 & 199.7 \\
Meets & 51.4 & 28.3 & 0.0 & 123.0 \\
Eggs & 43.7 & 24.5 & 0.0 & 101.0 \\
Milk and Dairy products & 212.5 & 136.2 & 0.0 & 570.7 \\
\hline
\end{tabular}

blood chemistry showed that factor 1 was positively related to age and HDL cholesterol, and negatively associated with triglycerides. Factor 2 was positively associated with creatinine and uric acid (Table 9). Factor 3 positively associated with body weight and negatively associated with GOT.

Intake of flavonoids and BMI did not show any significant association (Fig. 3a), but those who consumed more flavonoids belonged to the ideal range of BMI. Neither intake of isoflavonoids nor flavonoids was significantly associated with bone density (Fig. 3b).

\begin{tabular}{|c|c|c|c|c|}
\hline Categories & Spices & Foods & $\mathbf{N}$ & \\
\hline \multirow[t]{32}{*}{ Vegetables } & Umbelliferae & Carrot & 48 & 20.1 \\
\hline & & $\begin{array}{l}\text { Parsley } \\
\text { Others }\end{array}$ & & $\begin{array}{l}0.2 \\
0.3\end{array}$ \\
\hline & Cucurbitaceae & Cucumber & 46 & 55.2 \\
\hline & & Pumpkin & 28 & 20.8 \\
\hline & & $\begin{array}{l}\text { Wax gourd } \\
\text { Others }\end{array}$ & & $\begin{array}{l}3.8 \\
0.8\end{array}$ \\
\hline & Solanaceae & Eggplant & 39 & 38.0 \\
\hline & & Tomato & 44 & 65.0 \\
\hline & & Green pepper & & 8.4 \\
\hline & & Others & & 0.9 \\
\hline & Cruciferae & Radish & 42 & 30.8 \\
\hline & & Cabbage & 35 & 16.5 \\
\hline & & Komatsuna & 10 & 4.5 \\
\hline & & Chinese cabbage & 5 & 3.8 \\
\hline & & Broccoli & 9 & 3.0 \\
\hline & & Others & & 3.7 \\
\hline & Liliaceae & Onion & 40 & 19.6 \\
\hline & & Welsh onion & 37 & 6.1 \\
\hline & & Chinese chine & 9 & 1.4 \\
\hline & & Garlic & 20 & 0.4 \\
\hline & & Others & & 1.6 \\
\hline & Zingiberaceae & Ginger & 35 & 1.9 \\
\hline & & Myoga & 28 & 3.8 \\
\hline & Leguminosae & Kidney bean & 25 & 7.6 \\
\hline & & Bean sprout & 16 & 6.2 \\
\hline & & Green soybean & 12 & 3.7 \\
\hline & & Others & & 1.4 \\
\hline & Compositae & Lettuce & 22 & 7.5 \\
\hline & & Edible bardock & 13 & 3.0 \\
\hline & & Others & & \\
\hline & Chenopodiaceae & Spinach & 16 & 5.3 \\
\hline & Tiliaceae & Jew's marrow & 17 & 7.5 \\
\hline & Others & Other vegetables & 541 & 13.4 \\
\hline Nuls and seeds & Pedaliaceae & $\begin{array}{l}\text { Sesame seeds } \\
\text { Others }\end{array}$ & 23 & $\begin{array}{l}1.0 \\
1.0\end{array}$ \\
\hline \multirow[t]{5}{*}{ Tubes } & Araceae & Konnyaku & 24 & 10.7 \\
\hline & & Satoimo & 8 & 4.7 \\
\hline & Convolvulaceae & Sweet potato & 18 & 4.7 \\
\hline & Solanaceae & Potato & 41 & 27.4 \\
\hline & Dioscoreaceae & Yamanoimo & 6 & 5.6 \\
\hline
\end{tabular}


Table 6. Intake of Vitamins, Isoflavonoids and Flavonoids.

\begin{tabular}{lrrrr} 
& & & \multicolumn{2}{c}{ N=50 mg/day capita } \\
\hline Vitamins \&Polyphenols & Mean & S.D. & Min & Max \\
\hline Retinol & 0.37 & 0.63 & 0.02 & 3.59 \\
Carotenoid & 3.35 & 1.48 & 0.84 & 7.22 \\
Vitamin B1 & 1.25 & 0.4 & 0.8 & 3.39 \\
Vitamin B2 & 1.51 & 0.39 & 0.93 & 2.86 \\
Niacin & 18.8 & 5.5 & 7.9 & 31.8 \\
Vitamin C & 117 & 47 & 47 & 280 \\
Tocopherol & 21.15 & 5.48 & 9.4 & 31 \\
\hline Daidzein & 16.20 & 7.65 & 3.18 & 35.61 \\
Genistein & 23.27 & 11.25 & 4.62 & 52.12 \\
\hline Myricitrin & 0.01 & 0.01 & 0.00 & 0.06 \\
Rutin & 1.50 & 1.68 & 0.19 & 7.90 \\
Myricetin & 0.57 & 0.55 & 0.00 & 2.17 \\
Fisetin & 0.39 & 0.54 & 0.01 & 2.76 \\
Eriodictyol & 0.27 & 0.48 & 0.00 & 1.94 \\
Quercetin & 8.28 & 6.63 & 0.46 & 26.90 \\
Luteolin & 0.28 & 0.20 & 0.00 & 0.76 \\
Kaempferol & 4.91 & 2.84 & 0.84 & 12.82 \\
\hline
\end{tabular}

Table 7. Results of Principle Component Analysis.

\begin{tabular}{lccccc}
\hline \multicolumn{5}{c}{ Table 7. Results of Principle Component Analysis. } \\
\hline Vitamins \&Polyphenols & Factor 1 & Factor 2 & Factor 3 & Factor 4 & \multicolumn{1}{c}{ Factor 5 } \\
\hline Retinol & 0.718 & -0.170 & 0.383 & 0.071 & -0.335 \\
Carotenoid & 0.795 & -0.169 & 0.027 & -0.085 & 0.320 \\
Vitamin B1 & 0.439 & 0.313 & 0.040 & 0.135 & 0.420 \\
Vitamin B2 & 0.720 & 0.081 & 0.392 & -0.233 & -0.089 \\
Niacin & 0.583 & 0.137 & 0.105 & -0.424 & 0.113 \\
Vitamin C & 0.831 & 0.147 & -0.152 & -0.190 & 0.007 \\
Tocopherol & 0.432 & 0.626 & -0.257 & 0.083 & -0.056 \\
Daidzein & 0.094 & 0.872 & -0.401 & -0.027 & -0.085 \\
Genistein & 0.091 & 0.844 & -0.447 & -0.039 & -0.086 \\
Myricitrin & -0.071 & 0.657 & 0.665 & 0.275 & 0.089 \\
Rutin & 0.813 & -0.141 & -0.007 & 0.173 & -0.320 \\
Myricetin & 0.751 & -0.102 & -0.220 & 0.307 & 0.164 \\
Fisetin & 0.064 & -0.052 & 0.325 & -0.574 & 0.342 \\
Eriodictyol & -0.080 & 0.673 & 0.656 & 0.273 & 0.084 \\
Quercetin & 0.428 & -0.338 & 0.104 & 0.526 & -0.158 \\
Luteolin & 0.410 & -0.270 & -0.264 & 0.379 & 0.544 \\
Kaempferol & 0.800 & -0.027 & -0.036 & -0.217 & -0.242 \\
\hline Eigenvalue & 5.323 & 3.171 & 1.871 & 1.382 & 1.070 \\
Per.of var. & 31.3 & 18.7 & 11.0 & 8.1 & 6.3 \\
\hline cumulative & 31.3 & 50.0 & 61.0 & 69.1 & 75.4
\end{tabular}

Table 9. Association between Factors of Principle Component Analysis and Physical and Laboratory Data.

\begin{tabular}{|c|c|c|c|c|}
\hline Variable & Factor 1 & Factor 2 & Factor 3 & Factor 4 \\
\hline Age & $0.339 *$ & 0.193 & -0.050 & -0.060 \\
\hline Systolic Blood Pressure & -0.076 & 0.089 & 0.056 & 0.063 \\
\hline Diastolic Blood Pressure & -0.209 & -0.131 & 0.087 & 0.011 \\
\hline Height & -0.201 & 0.015 & 0.082 & 0.220 \\
\hline Weight & -0.215 & -0.090 & $0.302 *$ & 0.155 \\
\hline Body Mass Index & -0.137 & -0.086 & 0.274 & 0.045 \\
\hline Bone Density & -0.282 & -0.096 & -0.048 & 0.049 \\
\hline GOT & 0.239 & -0.009 & $-0.33 I^{*}$ & -0.070 \\
\hline GPT & 0.009 & -0.107 & -0.178 & 0.106 \\
\hline Serum total cholesterol & -0.003 & 0.143 & 0.079 & 0.061 \\
\hline HDL Cholesterol & $0.313 *$ & -0.092 & 0.068 & 0.027 \\
\hline Triglyceride & $-0.307^{*}$ & -0.004 & 0.202 & 0.017 \\
\hline Creatinin & -0.059 & $0.406 * *$ & -0.020 & -0.051 \\
\hline Uric Acid & -0.097 & $0.309 *$ & -0.155 & 0.261 \\
\hline
\end{tabular}

$y=77.4 x+2097$

$\mathrm{r}^{2}=0.244 \mathrm{n}=50$

$r=0.494 p<0.001$

Carotene $(\mu \mathrm{g} /$ day $)$

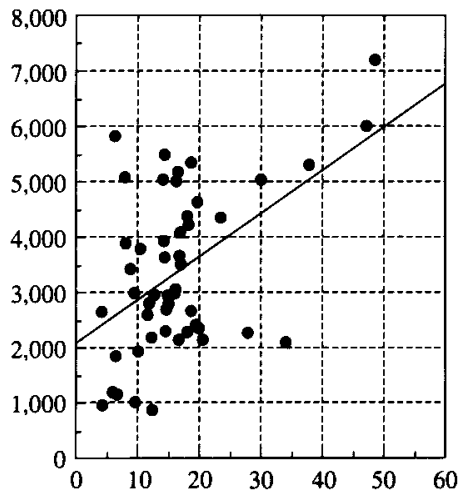

Flavonoids Intake (mg/day)
Table 8. Association between Factors of Principle Component Analysis and Food Categories.

\begin{tabular}{|c|c|c|c|c|}
\hline Variable & Factor 1 & Factor 2 & Factor 3 & Factor 4 \\
\hline Number of foods & $0.332 *$ & 0.047 & 0.237 & 0.067 \\
\hline Rice & 0.085 & 0.046 & -0.243 & -0.133 \\
\hline Bread & -0.193 & 0.100 & 0.109 & 0.176 \\
\hline Noodle & -0.068 & -0.141 & $0.363^{* *}$ & 0.017 \\
\hline Potatoes & $0.329 *$ & 0.059 & -0.106 & -0.043 \\
\hline Fat \& Oil & 0.042 & $0.313^{*}$ & -0.027 & $0.402 * *$ \\
\hline Legume & 0.078 & $0.800^{* *}$ & $-0.460 * *$ & -0.089 \\
\hline Fruits & 0.227 & 0.031 & 0.226 & $-0.509 * *$ \\
\hline G. Y. Vegetables & $0.579 * *$ & -0.045 & -0.236 & -0.138 \\
\hline Other Vegetables & $0.510^{* *}$ & 0.276 & 0.015 & -0.180 \\
\hline Fish & $0.439 * *$ & -0.042 & -0.041 & -0.232 \\
\hline Meat & 0.193 & -0.026 & 0.051 & 0.040 \\
\hline Egg & 0.244 & 0.005 & 0.226 & 0.010 \\
\hline Milk & -0.023 & -0.087 & $0.358^{*}$ & -0.206 \\
\hline
\end{tabular}

$\mathrm{y}=2.0 \mathrm{x}+84.3$

$\mathrm{r}^{2}=0.170 \mathrm{n}=50$

$r=0.412 p<0.001$

Vitamin C (mg/day)

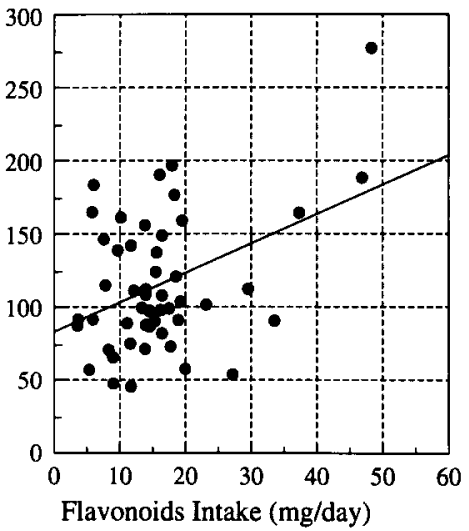

$y=0.2 x+14.2$

$r^{2}=0.362 n=50$

$r=0.602 \mathrm{p}<0.001$

Tocopherol (mg/day)

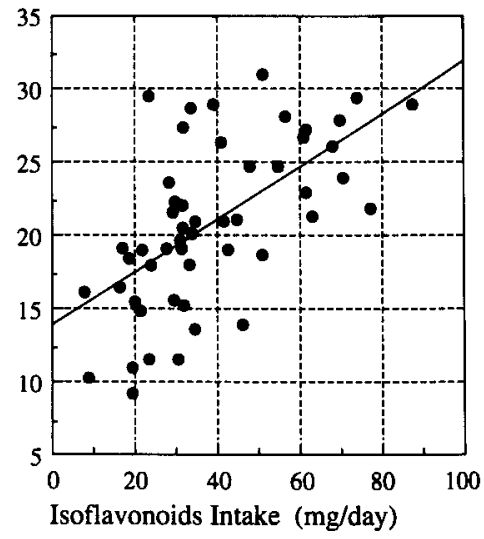

Figure 2. (ab) Association between flavonoid and carotene and vitamin C.

(c) Association between isoflavonoid and tocopherol intake. 

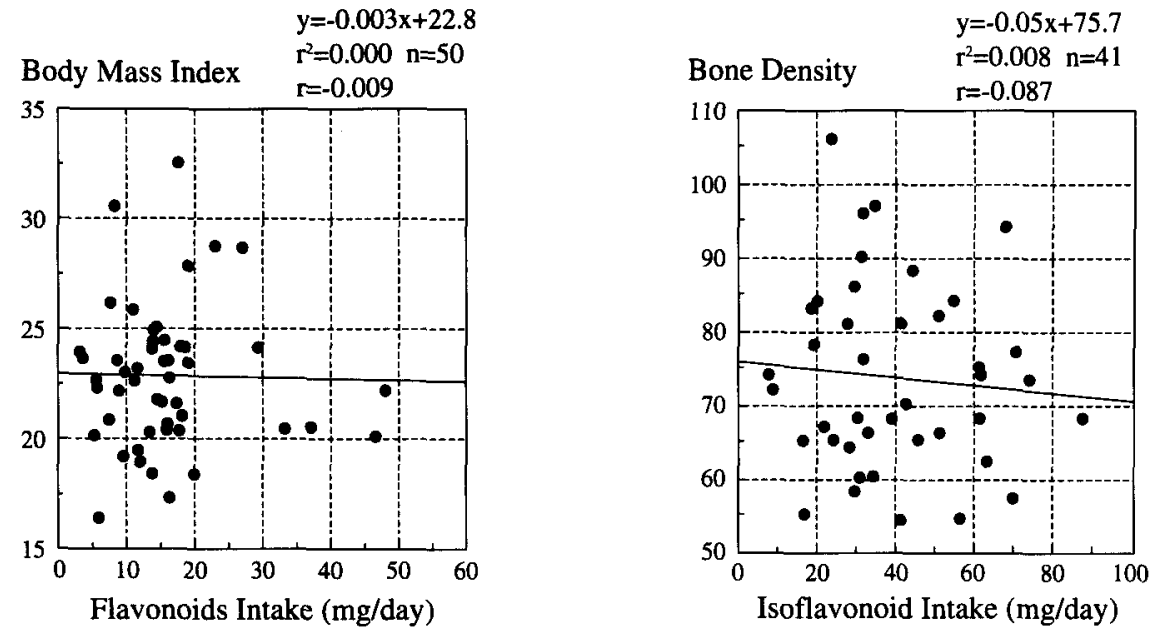

Figure 3. (a) Association between flavonoid intake and BMI.

(b) Association between isoflavonoid intake and bone density

\section{DISCUSSION}

The importance of non-nutrient phytochemicals for human health has been recognized ${ }^{9)}$.

In "I-City" of Iwate Prefecture, we have continued a nutritional survey for more than 15 years. The participants in the study were volunteers with high incentives for good nutrition, so the rates of nutrient intake satisfied the level of RDA for Japanese, except for calcium intake. SMR of cancer in "I-City" in 1990 was 0.93 , but that of cerebrovascular diseases was 1.40. ${ }^{10)}$. To understand the effects of phytochemical intake on human health, we tried to estimate the amount of intake of flavonoids in this area. This research also provides fundamental data for future study in different areas in Japan. Isoflavonoid intake was also estimated, because blood and urinary levels were very high compared to the Caucasians ${ }^{11}$. Data on the content of flavonoids and isoflavonoids in Japanese foods had been quite insufficient up to this point.

Hertog et al ${ }^{12)}$ determined the content of quercetin, kaempferol, myricetin, apigenin, and luteolin among $28 \mathrm{veg}-$ etables and 9 fruits by Reversed phase-HPLC with UV detection for estimating flavonoids intake from foods. Quercetin levels in the edible parts of most vegetables were generally below $10 \mathrm{mg} / \mathrm{kg}$ except for onions $(284-486 \mathrm{mg} / \mathrm{kg})$, kale (110 $\mathrm{mg} / \mathrm{kg}$ ), broccoli (30 mg/kg), French beans (32-45 $\mathrm{mg} / \mathrm{kg}$ ), and slicing beans (28-30 $\mathrm{mg} / \mathrm{kg}$ ). Kaempferol could only be detected in kale (211 mg/kg), endive (15-91 mg/kg), leeks (11-56 $\mathrm{mg} / \mathrm{kg}$ ), and turnip tops (31-64 mg/kg). The content of myricetin, luteolin, and apigenin was below the limit of detec- tion $(<1 \mathrm{mg} / \mathrm{kg}$ ) except for fresh broad beans $(26 \mathrm{mg} / \mathrm{kg}$ myricetin) and red bell peppers (13-31 mg/kg luteolin). Seasonal variability was low for most vegetables except leafy vegetables which have the highest flavonoid levels in summer.

Our data base is still preliminary, but it covers 40 food items and would cover $77 \%$ of flavonoid and isoflavonoid intake from vegetables, $99.5 \%$ from legumes, $58.4 \%$ from potatoes, and $43.5 \%$ from fruits included in this study. In the Zutphen Elderly Study, mean baseline flavonoids intake was $25.9 \mathrm{mg}$ per day ${ }^{2}$. The major sources of intake were tea (61\%), onions $(13 \%)$, and apples $(10 \%)$. Rimm et al $\left.{ }^{13}\right)$ reported the average intake of flavonoids of Americans was $20 \mathrm{mg} /$ day coming from black tea $(25 \%)$, onions $(25 \%)$, apples (10\%) and broccoli (7\%).

Japanese in our study consumed $16.22 \mathrm{mg}$ of flavonoids, which seemed to be less than Caucasians. Although catechin was not included in the present study, average tea consumption was $300 \mathrm{ml}$, in which the amount of flavonoids would be less than $3 \mathrm{mg}$. On the contrary, isoflavonoid intake was much higher than flavonoids. The isoflavonoid level found in Caucasians was reported to be low, but the Japanese level was very high ${ }^{11}$, although inter-individual differences were large.

The health effects of these chemicals are still under observation in Japan. These could be clarified by a nested-case control study in the large prospective study ${ }^{14)}$. The average intake of flavonoids was inversely associated with mortality from coronary heart disease and provided an explanation for $25 \%$ of the variance in coronary heart disease rates in the 16 cohorts of the Seven Country Study ${ }^{15}$ ). In multivariate analysis, intake of sat- 
urated fat $(73 \% ; \mathrm{p}=0.001)$, flavonoid intake $(8 \%, \mathrm{p}=0.01)$, and percentage of smokers per cohort $(9 \% ; \mathrm{p}=0.03)$ together explained $90 \%$ of the variance in coronary heart disease rates. These variables were independent of intake of alcohol and antioxidant vitamins. Average flavonoid intake may partly contribute to differences in coronary heart disease mortality across populations, but it does not seem to be an important determinant of cancer mortality.

The preventative properties of flavonoids are considered to be due to their strong antioxidant action ${ }^{16}$. Hertog et al. ${ }^{17}$ measured quercetin, kaempferol, myricetin, apigenin, and luteolin in the Zutphen Elderly Study. The relative risk of coronary heart disease mortality in the highest versus the lowest tertial of flavonoid intake was 0.42 (95\% CI; 0.2-0.88). After adjustment for various factors, the risk was still significant $(0.32$ (0.15-0.71)). Intake of tea, onions and apples as major sources of flavonoids was also inversely related to coronary heart disease mortality, but this association was weaker.

In some reports, however, such benefit was not confirmed. Rimm et al ${ }^{13)}$ reported that the upper/lower flavonoid intake quintile RR of non-fatal cardiac infarct (496 cases) was 1.08 (95\% CI; 0.81-1.43) among 34,789 males (40-75 y/o). Hertog et al ${ }^{17}$ found in a Welsh population of men in the Caerphilly Study, that men with the highest consumption of tea $>1.2 \mathrm{~L}$, or $>8 \mathrm{cups} / \mathrm{d}$ ) had an RR of 2.4 (95\% CI: 1.5-3.9) of dying by ischemic heart disease, during the follow-up period, compared with men consuming $<300 \mathrm{ml} / \mathrm{d}$ ( 2 cups $/ \mathrm{d}$ ). They considered that adding milk to tea destroyed the plasma antioxidant-raising capacity of tea. Interaction of these phytochemicals during cooking and with other biomolecules in the body are considered important.

The mechanism of the biological effects of different metabolites also need to be clarified. Manach et al ${ }^{18)}$ studied the dietary quercetin recovery in rat plasma and found conjugated derivatives of isorhamnetin, 3'-O-methylated form of quercetin in the plasma from the rats fed $0.25 \%$ quercetin. After deconjugation, the concentration of aglycones in the plasma reached $120 \pm 16 \mu$ mole/l, with an isorham-netin/quercetin ratio of about 5 . In bile and in urine, where the 4-oxo-flavonoid concentration were $378 \pm 42$ and $128 \pm 19 \mu \mathrm{mole} / \mathrm{L}$, respectively. Watanabe et al. ${ }^{19)}$ reported the metabolism of isoflavonoids after intake of $60 \mathrm{~g}$ soy bean powder in men, and found that the blood level reached $4.0 \mu$ mole $/ \mathrm{L}$ which is much higher than that of the endogenous 17-beta estradiol level. This indicated complicated interaction of food-derived phytochemicals (factors) and body constituent biomolecules taking place inside the body.

Beta-carotene was once considered to be a representative antioxidant for cancer prevention, but interaction with other chemicals should occur. The distinction of various food factors should be determined as to whether they are real or surrogate markers for epidemiological study ${ }^{9}$.

\section{REFERENCES}

1) Hertog MGL, Feskens EJM, Hollman PCH, Katan MB, Kromhout D. Dietary antioxidant flavonoids and risk of coronary heart disease: the Zutphen Elderly Study. Lancet 1993; 342: 1007-1011.

2) Frankel EN, Kanner J, German JB, Parks E, Kinsella JE. Inhibition of oxidation of human low-density lipoprotein by phenolic substances in red wine. Lancet 1993; 341: 454-57.

3) Frankel EN. Nutritional benefits of flavonoids. In; Food Factors for Cancer Prevention, Ohigashi H, et al (eds.) Springer-Verlag, Tokyo, 1997, pp613-616.

4) Watanabe S, Koessel S. Colon cancer; An approach from molecular epidemiology. J Epidemiol 3: 47-61, 1993.

5) Adlercreutz H, Goldin BR, Gorbach SL, Hockerstedt KAV, Watanabe S, Hamalainen EK, Markkanen MH, Makela TH, Wahala KT, Hase TA, Fotsis T. (1995) Soybean phytoestrogen intake and cancer risk. J Nutr 125: 757s-770s

6) Shimoi K. Evaluation of food-derived phytoflavonoids as antioxidant in body. Monbusho Kagaku Kenkyu Report 1995-6, 1998

7) Hertog MGL, Hollman PCHI Venema DP. Optimization of a quantitative HPLC determination of potentially anticarcinogenic flavonoids in vegetables and fruits. J Agric Food Chem 1992; 40: 1591-1598.

8) Adlercreutz H, Fotsis $T$, Watanabe $S$, Lampe J, Wahala $\mathrm{K}$, Makela T, Hase (1994) Determination of lignans and isoflavonoids in plasma by isotope dilution gas chromatography-mass spectrometry. Cancer Detect Prev Med 18:259-271.

9) Watanabe S, Kimira M, Sobue T. Cancer prevention: approaches from epidemiology, In; Ohigashi $\mathrm{H}$, et al (eds.), Food Factors for Cancer Prevention, Springer Verlag, Tokyo, Berlin, Heiderberg, pp. 3-8, 1997

10) Statistical Bureau, Ministry of Health and Welfare, Vital Statistics 1995, Ministry of Health and Welfare

11) Adlercreutz H, Markkanen H, Watanabe S. (1993). Plasma concentrations of phyto-oestrogens in Japanese men. Lancet 342: 1209-1210.

12) Hertog MGL, Hollman PCH, Katan MB. Content of potentially anticarcinogenic flavonoids of 28 vegetables and 9 fruits commonly consumed in the Netherlands. $J$ Agric Food Chem 1992; 40: 2379-2383.

13) Rimm EB, Katan MB, Archerio A, Stampler MJ, Willett WC. Relation between intake of flavonoids and risk for chronic heart diseases in male health professionals. Ann Intern Med 1996; 125: 384-389.

14) Watanabe $S$, Large-scale, population-based prospective studies in Japan. Eur J Cancer 1993; 29A: 2305-2314,

15) Hertog MGL, Kromhout D, Aravanis C, Blackburn H, 
Buzina R, Fidanza F, Giampaoli S, Jansen A, Menotti A, Nedeljkovic S, Pekkarinen M, Simic BS, Toshima H, Feskens EJM, Hollman PCH, Katan MB. Flavonoid intake and long-term risk of coronary heart disease and cancer in the seven countries study. Arch Intern Med 1995; 155: 381-386.

16) Ho C-T. Antioxidant properties of plant flavonoids. In; Food Factors for Cancer Prevention, Ohigashi H, et al (eds.) Springer-Verlag, Tokyo, 1997, pp593-597.

17) Hertog MGL, Sweetnam PM, Fehily AM, Elwood PC, Kromhout D. Antioxidant flavonoids and ischemic heart disease in a Welsh population of men: the Caerphilly Study. Am J Clin Nutr 1997; 65: 1489-1494.

18) Manach C, Texier O, Regerat F, Agullo G, Demigne C, Remesy C. Dietary quercetin is recovered in rat plasma as conjugated derivatives of isorhamnetin and quercetin. $\mathrm{J}$ Nutr Biochem 1996; 7: 375-380.

19) Watanabe S, Yamaguchi M, Sobue T, Takahashi T, Miura T, Arai Y, Mazur W, Wahala K, Adlercreutz H. Pharmacokinetics of soybean isoflavones in plasma, urine and feces after single ingestion of kinako, a soy protein product, by men. J Nutr (in press). 